Valdaliso Casanova, Teresa.

\title{
El concepto de "Estación Creativa"; un proyecto reticular basado en el proceso creativo compartido.
}

\section{The concept of "Creative Station"; a network project based on a creative shared process.}

TIPO DE TRABAJO:

Comunicación virtual.

PALABRAS CLAVE:

Red, laboratorio, estación, creativa, metrópolis.

KEY WORDS:

Network, workshop, creative, station, metrópolis.

\section{RESUMEN.}

A partir de la idea de metrópolis como un nudo de conexiones donde la localización geográfica del núcleo central se propone temporal y de carácter itinerante, quedando en ocasiones desplazado a la periferia, se plantea un proyecto de intercambio creativo cuya estructura de actuación viene definida en modo reticular, fomentando la regeneración y reutilizo de espacios en desuso, o bien utilizando nuevos espacios situados en territorios que no disfrutan de la consideración que merecen.

La descentralización de los lugares de creación y divulgación artística implica la reconfiguración de una identidad territorial perdida que, presentándose como irrecuperable, decide reinventarse a partir de un nuevo concepto de espacio de fusión y fruición, un punto de encuentro y de adición de diversas realidades que se encuentran en un ámbito local, procedentes a su vez de otros ámbitos locales, pertenecientes todos a una misma red de intercambios.

Dicha idea se materializa a partir del concepto de "Estación Creativa", desarrollado teóricamente por los italianos Paolo Rosa (artista y docente) y Andrea Balzola (dramaturgo y docente), siendo llevada a cabo por primera vez en el Spazio MIL de Sesto San Giovanni (Milán) en los meses de Abril-Mayo de 2016.

Creativos, artistas y estudiosos de diversas disciplinas actuaron como agentes culturales a la vez que sociales, a partir de una perspectiva de arte político entendiendo la polis como la extensión de lugar en donde todos cohabitamos. Activadores de imaginarios compartidos, incluyeron al visitante como parte integrante del proceso creativo, un proceso basado en la formación, la investigación y la producción. Donde el aura no pertenece ya a la obra de arte, sino a la experiencia artística común.

http://www.spaziomil.org/evento/stazione-creativa-2/

ABSTRACT.

Considering the idea of the metropolis as a knot of connections where the geographical location of the central core plays a temporary and itinerant role, being frequently displaced to the periphery, a project of creative exchange is proposed, which acting structure is defined in a reticulated way, promoting the regeneration and I re-utilization of spaces in disuse, or using new spaces placed in territories that don't receive the consideration that they deserve. 
The decentralization of the creative places and artistic divulgation, implies the reconfiguration of a lost territorial identity that, appearing irrecoverably, decides reinvent itself from a new concept of space of merging and fruiting, a point of meeting and of addition of diverse realities that can be found in a local area, proceeding at the same time from other local areas, all of them belonging to the same exchanging network.

This idea is materialized by the concept of " Creative Station ", developed theoretically by Italians Paolo Rosa (artist and teacher) and Andrea Balzola (playwright and teacher), that's been realized for the first time in the Spazio MIL, in Sesto San Giovanni (Milan) in April - May, 2016.

Creatives, artists and researchers of diverse disciplines operated as cultural and social agents, from a perspective of political art where the polis means the extension of place where we all live together. Activators of a shared imaginary, they include the visitor as integral part of the creative process, a process based on the formation, the investigation and the production. Where the aura does not belong to the work of art any more, but to the artistic common experience.

http://www.spaziomil.org/evento/stazione-creativa-2/

\section{CONTENIDO.}

\section{Introducción.}

El concepto moderno de metrópolis ha ido evolucionando desde la revolución industrial hasta la actualidad por motivos, fundamentalmente, económicos y demográficos. La tendencia del siglo XXI define el área metropolitana como una gran ciudad cuyo sistema administrativo funciona como un ente autónomo local, a partir del cual se gestionan diversos territorios circundantes, los cuales en un principio fueron destinados a acoger la industria, los servicios, el comercio o incluso las ciudades dormitorio, y cuyo futuro se dirige hacia una idea de un territorio expandido que supone un cruce de caminos entre lo global y lo local, donde habitar e interactuar con una serie de eventos promovidos a través de una estructura reticular. Dicho concepto, en su avance, proclama redefinir una nueva dimensión, marcada sobre todo por dos factores característicos del mundo contemporáneo: la tendencia a la totalización a través de los medios de comunicación y, a causa de los mismos, el artificio de la ciudad de neón (Fiorani en Folci, Rosa, 2012, p.84). La solución propuesta será la de utilizar las posibilidades que ofrecen dichos factores para la creación de nuevos espacios que supongan puntos de encuentro de imágenes, sonidos, luces, historias; escenarios funcionales y emotivos, sonidos provenientes del ritmo de la vida urbana; gestos, eventos, encuentros donde se sobrepase la diferencia entre dentro y fuera, y el sentido de lo global se entrelace con el de local, en una constante mutación de la modalidad de disfrute del espacio y una reconfiguración de la propia identidad, cabalgando con cierta velocidad, proporcional a la de la metamorfosis del flujo urbano al cual están conectadas.

\section{El concepto de "Estación Creativa".}

É necesario impegnarsi, su un piano più diretto di política culturale, per valorizazare gli ambienti territoriali, gli habitat in cui agire. Anche il territorio creativo, geográficamente localizzato, ha bisogno di una vera infrastruttura organizzata. Così come la rete dei trasporti necesita di nodi di scambio e la rete virtuale si avvale di siti chiave di informazione, aggregazione e progettazione, queta infrastruttura ha bisogno di luoghi concreti in cui identificarsi e svilupparsi, organizzabili in una rete diffusa su un territorio più ampio, estendibile idealmente al territorio globale: questi luogi posono configurarsi come stazioni creative [...] (Balzola, Rosa, 2011)

Es necesario empeñarse sobre un plano más directo de política cultural para valorar los ambientes territoriales, los hábitats en los cuales actuar También el territorio creativo, geográficamente localizado, necesita una verdadera infraestructura organizada. Así como la red de transportes necesita nudos de intercambio y la red virtual se sirve de sitios claves de información, agregación y proyectación, esta infraestructura necesita lugares concretos en los cuales identificarse y desarrollarse, organizados en una red difundida en un territorio más amplio, extendible ideológicamente al territorio global: estos lugares pueden configurarse como estaciones creativas [...] (trad.a.)

La idea de la estación creativa (stazione creativa) fue desarrollada por el desaparecido artista Paolo Rosa (Rímini 1949-Corfú 2013), miembro fundador del histórico grupo artístico milanés Studio Azzurro ${ }^{1}$, en colaboración con el teórico Andrea Balzola ${ }^{2}$. El concepto

\footnotetext{
${ }^{1}$ El grupo Studio Azzurro fue fundado en Milán en 1982 por tres artistas: Paolo Rosa, Fabio Cirifino y Leonardo Sangiorgi, Su producción artística se ha centrado, a lo largo de más de 30 años, en la exploración de los lenguajes que nacen de las nuevas tecnologías y que han producido las grandes mutaciones del siglo XX. Partiendo de una práctica colaborativa, basan su investigación en el intercambio de conocimientos, proponiendo experiencias que integran tanto a otros creadores como al público, huyendo del elitismo y de la especulación
} 
nació a finales de los años 90, periodo en que comenzaron a fraguar el proyecto de escribir conjuntamente un libro que contuviese varios conceptos e ideas comunes a ambos autores.

Dicho libro fue publicado en 2011 (Feltrinelli ediore, Milán) con el titulo L'arte fuori di sé ${ }^{3}$ (El arte fuera de sí), un manifiesto para la era tecnológica, escrito a cuatro manos. Con la premisa de recuperar cierta generosidad "desperdigada", asociándose en red y compartiendo así sabiduría y experiencia, proponen a los artistas "salir de sí mismos" asumiendo la función de catalizadores de energías creativas. En su manifiesto afirman que es necesario repensar y replantearse la función del artista, lo cual supone salirse de los límites preestablecidos, con el fin de "constituir nuevos paradigmas para comprender ciertos aspectos innovadores y cambiantes, y proponer nuevas modalidades para vehicular el diálogo entre las personas". Siguiendo la línea teórica de Derrida, defienden que el creativo debe ser anacrónico y un inadaptado a su tiempo, o de lo contrario no llevará a cabo su función, no innovará nunca. Proclaman un "artista político", en relación a los espacios y las prerrogativas de la polis; un artista que promueve la interlocución entre el territorio y la comunidad, investigando el impacto de los diversos factores que cohabitan en la red urbana. Bajo este punto de vista, la estación creativa se define como un cruce de disciplinas y competencias que no persigue juntar artistas en busca de un lugar expositivo y efímero para la propia obra, sino activadores y actores que produzcan in situ, y que provoquen situaciones (laboratorios, encuentros, performances...) donde fomenten una creación común en relación a la comunidad local, mezclada con visitantes provenientes de otras realidades.

Por parte de Paolo Rosa podemos encontrar vestigios de la idea de laboratorio colaborativo en las intervenciones realizadas en los años 70 por parte del grupo Laboratorio di Comunicazione Militante del cual formaba parte. En plena vorágine socio-educativa, tras la revuelta del 69, un grupo de artistas italianos ocupó la antigua iglesia de San Carpoforo (en el barrio de Brera, Milán) con la finalidad de llevar a cabo una serie de laboratorios enfocados a la ciudadanía y en especial a los jóvenes, ideados para concienciar a la sociedad acerca de la manipulación a la cual estaban siendo sometidos por parte de los medios de comunicación de masas. La idea era la de dar "armas" a los participantes para desenmascarar los trucos y recursos que eran comúnmente utilizados para tergiversar las noticias. Realizaban laboratorios itinerantes y exposiciones en las cuales mostraban los resultados. La dimensión más valiosa de dichas iniciativas no residía en el aspecto formal de la obra si no en lo que ésta generaba relacionalmente con las personas que dialogaban con los artistas. En los años 70 aún no se hablaba como hoy de la libertad de comunicación, ni de arte público, ni de involucrar al espectador en el proceso artístico, por ello el Laboratorio supuso una revuelta artística de carácter investigador y de inquietudes político-sociales, que se adelantó a su tiempo. La crisis económica y social de la globalización provocó la necesidad de un arte que, dentro de su marginalidad, volviera a ser el centro de un proceso de crítica y transformación a través de las prácticas participativas. Así mismo, los fundadores del Laboratorio redactaron varios manifiestos expresando su punto de vista, con títulos como: Cultura metropolitana e arte borghese (Cultura metropolitana y arte burgués) (Valdaliso, López, 2015).

De hecho el grupo artístico fundado la década posterior por Paolo Rosa, Studio Azzurro, ha mantenido desde el principio y durante 36 años dicho carácter colaborador, representado en lo que ellos llaman una "bodega del arte" donde se reúnen jóvenes y adultos, expertos y futuros artistas, para compartir el proceso creativo; los conocimientos y los resultados. Por otra parte, para los artistas del Studio siempre ha tenido fundamental importancia la función de la "obra participativa", partiendo del concepto de "obra abierta" que formulaba Eco, invitando al espectador a interactuar con ella y con el resto de participantes para crear una nueva versión de la obra, localizada en el aquí y ahora.

En el momento de su repentino fallecimiento en 2013, Rosa llevaba años trabajando en el proyecto de la Fabbrica del Vapore ${ }^{4}$ con Studio Azzurro, de cuya asociación era presidente, y que consideraba una potencial estación creativa. Balzola por su parte había creado varias asociaciones con la finalidad de desarrollar laboratorios permanentes de ideas y experimentaciones creativas, ya sea en el campo artístico, en el ámbito del teatro y la experimentación multimedia, como en el campo de la discapacidad mental.

del mercado del arte. Del bagaje adquirido en la práctica de la creación común, nace una predisposición al diálogo, un interés por considerar a su interlocutor como una fuente de sabiduría y un co-autor del proceso creativo. (Valdaliso, López, 2016)

${ }^{2}$ Balzola es dramaturgo y docente universitario, especializado en las artes escénicas multimediales.

${ }^{3}$ www.facebook.com/Larte-fuori-di-s\%C3\%A9-250766651602459

${ }^{4}$ La Fabbrica del vapore se encuentra cerca del Cementerio Monumental de Milán. Ex fábrica de locomotoras Carminati y Toselli, la estructura fue convertida en un espacio polivalente que hoy acoge eventos, performances y conciertos. Reestructurada en 2002 con la idea de albergar laboratorios creativos, el ayuntamiento cedió en alquiler sus espacios a 15 asociaciones y colectivos que actúan en diversos sectores: Polifemo (fotografía), Docva (archivo de documentación de artes visuales), Show Biz (producción televisiva), Ariella Vidach (danza y tecnología), Studio Azzurro (producciones artísticas audiovisuales), Viafarini y Careof (promoción de la investigación artística) One Off (material para el diseño y la arquitectura), etc. Paolo Rosa fue presidente de la asociación FdvLab (Fabbrica del vapore Lab) hasta su fallecimiento; afirmaba que pretendían ser una "estación creativa" para jóvenes artistas pero se "viajaba a una lentitud exasperante"; cuando en otras ciudades europeas solían esperar 2 ó 3 años para poder realizar un proyecto, en Milán habían pasado 15. La última nave acondicionada, la nave central llamada "la Cattedrale", funciona desde 2012 como espacio polivalente y cafetería. En el segundo piso de la nave Liberty donde se encuentra Studio Azzurro hay una residencia de artistas jóvenes, quienes participan temporalmente en alguno de los laboratorios. Tras sufrir cierto abandono y la carencia de una adecuada gestión por parte del ayuntamiento de Milán, la mayor parte de los colectivos han abandonado las instalaciones, y los espacios adyacentes al de Studio Azzurro están actualmente a la espera de nuevas concesiones. 
En cierta manera, la idea de la estación creativa se inspiró físicamente en los proyectos de conversión de las estaciones decadentes en museos de arte contemporáneo (como en Paris, Berlín, Montevideo) y en centros culturales, y conceptualmente en el hecho de que la estación es un lugar de parada temporánea, de intercambio, un cruce de caminos, desde donde parten y a donde llegan viajeros y con ellos sus ideas, extendidas a las practicas creativas; un lugar rizomático como lo definiría Deleuze.

En una reciente conversación con Andrea Balzola (conversación entre la autora de este texto y Balzola, en marzo de 2017), el dramaturgo señalaba que su intención era la de recuperar áreas abandonadas, antiguas fábricas o edificios públicos, situados en zonas como el municipio de Sesto San Giovanni, donde vivía Paolo Rosa. La idea era la de crear, con la ayuda de algunos amigos artistas, estudiosos y antiguos alumnos, una nueva Bauhaus Multimedia donde poder transferir y re-organizar la escuela de Nuevas Tecnologías de la Accademia di Belle Arti di Brera (Milán) que había sido creada por Paolo Rosa. Debido a varios inconvenientes de carácter burocrático, económicos y a ciertas desavenencias con colegas y directivos, comprendieron la imposibilidad de llevar a cabo la iniciativa dentro del circuito institucional. Decidieron intentar llevar a cabo el proyecto con otro tipo de realidades fuera del sistema educativo público, si bien a diferencia de las escuelas privadas, la idea de la estación creativa convertida en escuela Bahaus preveía involucrar otras realidades innovadoras y dinámicas, ya presentes en el territorio. Desgraciadamente Rosa no tuvo tiempo de llevarlo a cabo. Es por esto que la primera estación creativa fue desarrollada en su barrio, en Sesto San Giovanni, y fue dedicada a su memoria.

\section{La primera Estación Creativa en el Spazio MIL.}

La Città metropolitana de Milán fue constituida el 8 de abril de 2014 en la provincia de Milán, entrando así en vigor la Ley 56/2014 (art.12). El primer Consejo metropolitano fue elegido en septiembre de ese mismo año, siendo en plena asunción de patrimonio, personal y funciones el 1 enero 2015. Dentro del marco de la ciudad metropolitana, el estatuto declara el compromiso de desarrollar una mejor cultura de gobierno a través de la experiencia administrativa de los ayuntamientos del territorio, portadores de historias y tradiciones, en un cuadro integrado y policéntrico que respete la identidad y valore la participación.

El ayuntamiento de Sesto San Giovanni, situado a seis millas del centro de Milán, forma parte de la ciudad metropolitana. En el siglo XX supuso la sede de una de las más importantes concentraciones industriales de Italia, con establecimientos metalúrgicos, electrónica y electrotécnica, así como la industria textil y la producción editorial y de papel.

Este fue el municipio en el que vivió durante más de tres décadas el artista Paolo Rosa.

La primera Estación Creativa se desarrolló en los meses de abril y mayo de 2016 en el Spazio MIL, en dicho municipio. La asociación tiene sus instalaciones en una antigua fábrica de 3000 metros cuadrados, un lugar donde conviven el diseño, el teatro y la música. Un museo permanente donde realizar co-working, comer en su restaurante o pasear por el parque que lo circunda, un espacio rehabilitado.en una zona industrial en desuso. La elección del lugar fue, sin duda, influida por la necesidad de rendir homenaje al impulsor de la idea, y fue realizada en tal periodo con motivo de la exposición retrospectiva que se estaba celebrando contemporáneamente en el Palazzo Reale de Milán (Immagini sensibili, 9 abril - 4 septiembre 2016).

Coordinada conjuntamente por el Spazio MIL y por Osvalda Centurelli de Studio Azzurro, la estación fue promovida por el ayuntamiento de Sesto San Giovanni y por la fundación Carisbo. La iniciativa involucró a artistas, teóricos, estudiantes y demás participantes de todas las edades, del propio barrio y de otras localidades, incluso a nivel internacional, dirigiéndose a aquellos que "creen en la posibilidad de una ciudad metropolitana alimentada por lugares creativos".

Celebrada del 9 de abril al 12 de mayo, acogió 15 workshops didácticos dirigidos por 40 colaboradores entre artistas, docentes y expertos en el ámbito de la comunicación, provenientes de diversas regiones italianas. Todas las obras producidas y las videodocumentaciones de los workshop fueron presentados en la fiesta de clausura con la participación de casi medio millar de asistentes.

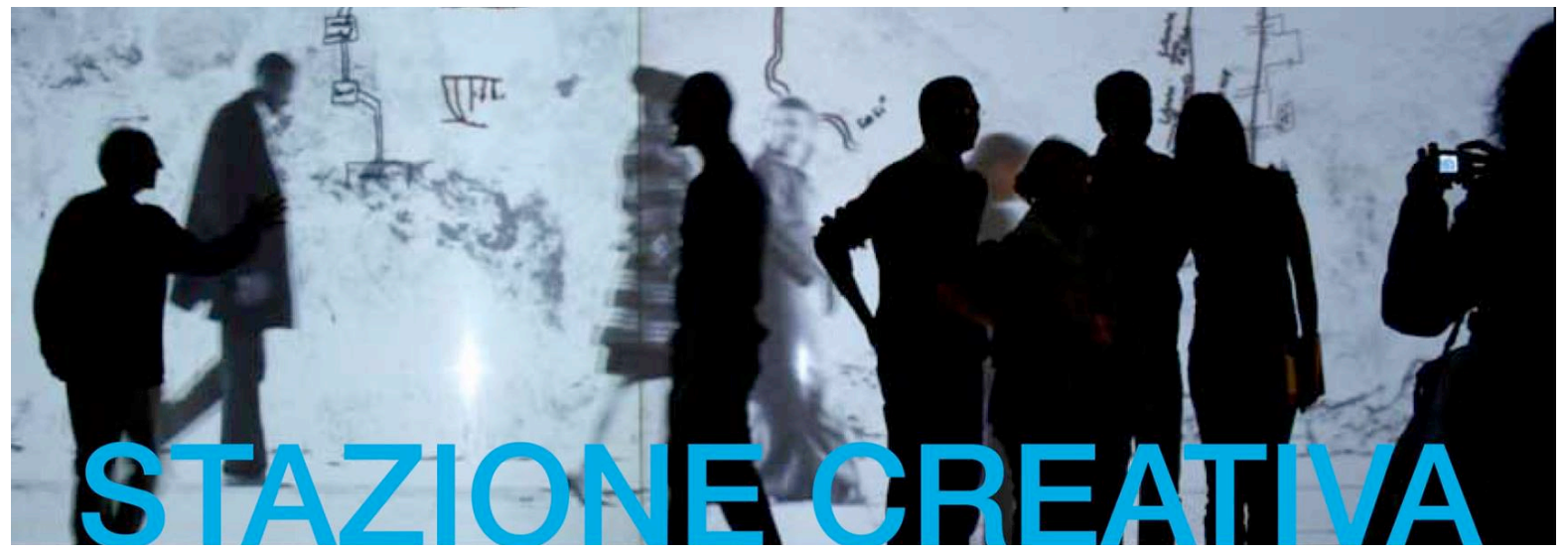

Imagen promocional de la Estación Creativa, perteneciente a una obra interactiva de Studio Azzurro, Sensible Map (2008). 
Inaugurada por Pompeo Martelli, director del Museo Laboratorio della Mente (Roma) y la artista Carol Pilar, comenzó con una conexión en streaming con el Palacio Real de Milán, donde acababa de inaugurarse la exposición retrospectiva de Studio Azzurro.

El programa de la primera Stazione Creativa comprendía laboratorios de acceso libre, encuentros bajo inscripción (gratuitos) y eventos performativos.

Los laboratorios fueron realizados por:

- Nucleo X, colectivo milanés cuya una trayectoria está marcada por el trabajo colectivo, conducido por el "arch-tista" (arqueólogo) Giulio Calegari y documentado por el filmmaker Giuseppe Baresi, el grupo proponía un punto de encuentro para recoger la materia prima necesaria para generar una "transmutación poética". Con el título: Accostarsi al territorio (Acercarse al territorio), pretendían acercarse a su memoria histórica a través de la realidad contemporánea, apoyados en el concepto de "geografía como teatro".

- Dissoi Logoi, suono e archeologie (sonido y arqueologías), conducido por el artista sonoro Alberto Morelli, proponía la creación de un objeto sonoro y la búsqueda de lugares resonantes en un espacio (Spazio MIL) que anteriormente fue un lugar de producción.

- $\quad$ AVES Project, ideado por Tullio Brunone, artista multimedia. El grupo experimentaba a partir de las dinámicas ligadas al hábitat, monitoreando el espacio, dialogando, mapeando. El material recogido fue elaborado utilizando lenguajes contemporáneos como el video, la fotografía, la gráfica y la instalación, para construir una narración que reflexionaba acerca de la forma del propio tiempo.

- DiStUrB, con el título de "Profezia", el laboratorio conducido por Francesca Capasso, Franco Cipriano, Antonio Davide, Matter, Pier Paolo Patti y Ciro Vitale, contemplaba una crítica profética del contemporáneo de carácter performativo. Material visual, verbal, sonoro y gestual se mezclaba en la representación de un "corpus imaginativo" donde una luz es sombra de otra luz, una señal es custodia de una ausencia, una imagen es un espacio, un mapa de la memoria que excede en el tiempo; un theatrum profético.

- Condominio 42, conducido por Davide Sgalippa, Alice Casalini, Gabriele Casiraghi, Carlotta Marabelli, Isabella Montan, Federica Rebaudengo, Luis Rosignoli y Federica Valiante, se trataba de un work in progress enfocado a la realización participativa de una instalación interactiva dedicada a un edificio histórico y controvertido situado en el corazón de Milán.

Se realizaron tres encuentros:

- La formazione come stazione creativa; semi per una rigenerazione (La formación como estación creativa, semillas para una regeneración). Workshop teórico-práctico organizado por Andrea Balzola en colaboración con Enya Daniela Idda, exponía una visión panorámica de las ideas, obras y la realidad de la innovación creativa y tecnológica, enfocadas a la regeneración del sistema educativo, con una conclusión final en la elaboración de haikuvideo.

- The map is not the territory (El mapa no es el territorio), encuentro conducido por Gabriele Donini , Anna Gialluca y Pietro Parisi (diseñadores), el objetivo era el de explorar y construir nuevos imaginarios del territorio de Sesto San Giovanni a partir de fotografías tomadas a través de mapas digitales para llegar a conformar artefactos imprevisibles.

- Arti migranti/arti accoglienti (Artes inmigrantes/artes acogedoras). Conducido por el diseñador Enzo Manzini, suponía un encuentro de trabajo sobre las problemáticas actuales y la potencialidad de las prácticas artísticas como instrumentos de inclusión social. La temática se afrontó a través de performances y lecciones teóricas.

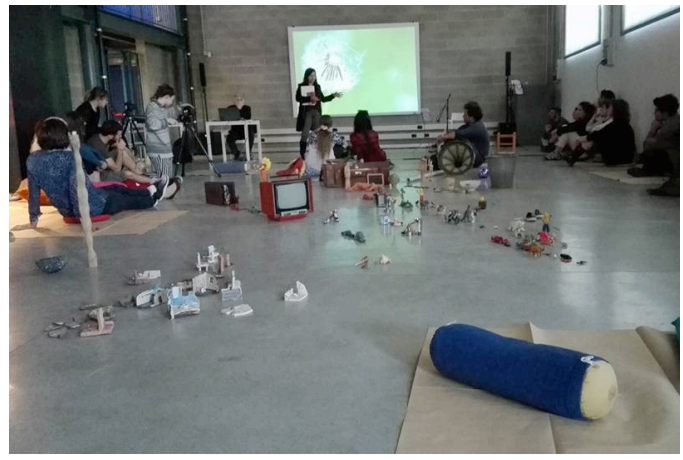

Laboratorio de Haikuvideo conducido por Balzola.

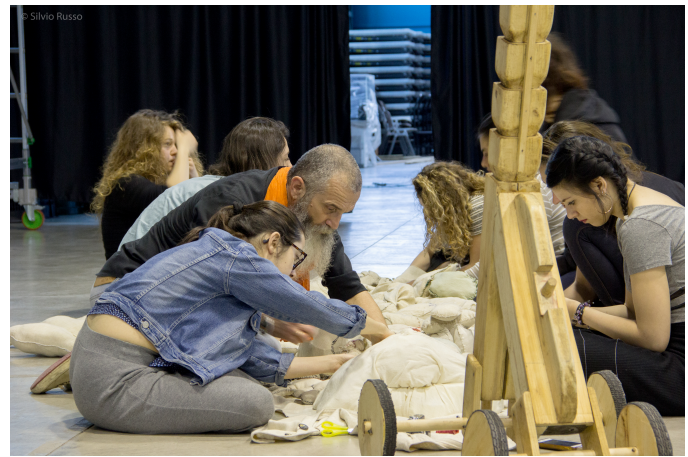

Francesco Marelli conduciendo "Trascinarsi il lavoro".

En el evento final confluyeron instalaciones interactivas, proyecciones audiovisuales, performances, música, danza y encuentros con los artistas. Una gran fiesta para compartir los resultados de los diferentes procesos creativos in progress. 


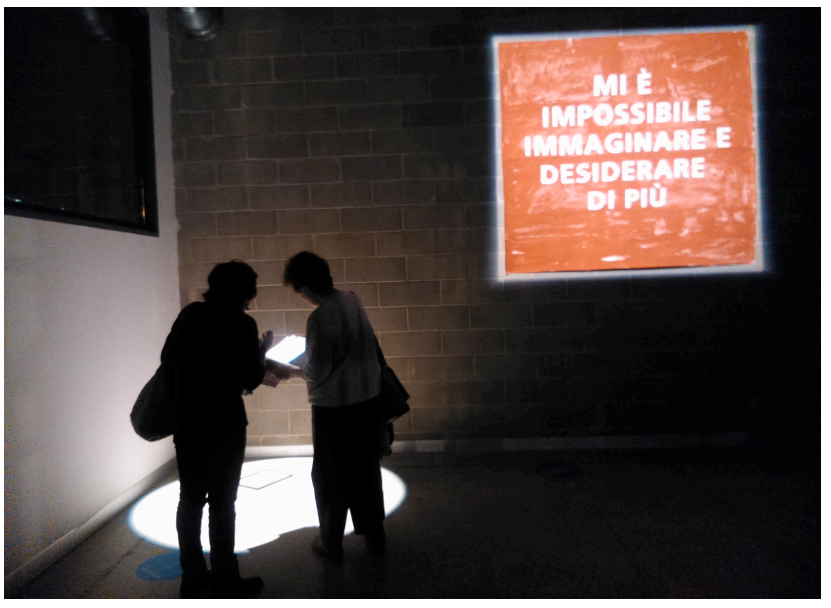

Performance video-musical del evento de clausura.

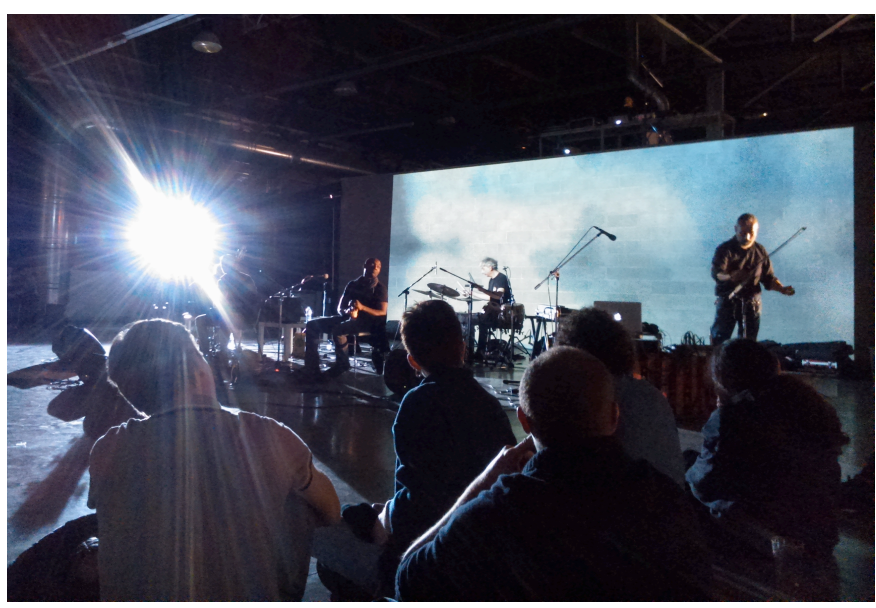

Obras en exposición en el evento de clausura.

\section{FUENTES REFERENCIALES.}

BALZOLA, A.; ROSA, P. (2011). L'arte fuori di sè. Un manifesto per l'età post-tecnologica. Feltrinelli editore, Milán.

ECO, U. (2006). Opera aperta. Forma e indeterminazione nelle poetiche contemporanee. RCS Libri S.p.A./Tascabili Bompiani, Milán.

LABORATORIO DI COMUNICAZIONE MILITANTE (1977). L'arma dell'immagine. Esperimenti di animazione sulla comunicazione visiva. Gabriele Mazzotta Editore, Milán.

FOLCI M., ROSA P. (2012), E manu capere. Sedici lezioni strane a Brera. Scalpendi Editore, Milán.

REBECCHI, M. "Sull'interattività. Conversazione con Pietro Montani." [en línea], en Alfabeta2, el 7 de diciembre de 2014. Disponible en www.alfabeta2.it/2014/12/07/sullinterattivita-conversazione-pietro-montani [consultado el 10 de abril de 2015]

VALDALISO, T; LÓPEZ, P. (2015). "El Laboratorio di Comunicazione Militante como ejemplo de estrategia social colaborativa." En las actas del congreso Metáforas de la multitud III Congreso Internacional Estética y Política. Valencia, 11-13 de noviembre de 2015. Editorial Universitat Politécnica de Valencia.

VALDALISO, T; LÓPEZ, P. (2016). Studio Azzurro: Tres décadas de experimentación en la creación audiovisual y los espacios sensoriales. Tesis defendida en la UPV de Valencia. 